\title{
Observational Evidence Linking Interstellar UV Absorption to PAH Molecules
}

\author{
Avi Blasberger ${ }^{1}$, Ehud Behar ${ }^{1,2}$, Hagai B. Perets ${ }^{1}$, Noah Brosch ${ }^{3}$, and Alexander G. G. M. Tielens ${ }^{4}$ \\ ${ }^{1}$ Department of Physics, Technion, Israel \\ ${ }^{2}$ Department of Astronomy, University of Maryland, College Park, MD, USA \\ ${ }^{3}$ The Wise Observatory and School of Physics and Astronomy, Tel Aviv University, Israel \\ ${ }^{4}$ Leiden Observatory, Leiden University, The Netherlands \\ Received 2016 November 7; revised 2017 January 4; accepted 2017 January 20; published 2017 February 17
}

\begin{abstract}
The $2175 \AA$ UV extinction feature was discovered in the mid-1960s, yet its physical origin remains poorly understood. One suggestion is absorption by polycyclic aromatic hydrocarbon (PAH) molecules, which is supported by theoretical molecular structure computations and by laboratory experiments. PAHs are positively detected by their 3.3, 6.2, 7.7, 8.6, 11.3, and $12.7 \mu \mathrm{m}$ IR emission bands, which are specified by their modes of vibration. A definitive empirical link between the $2175 \AA$ UV extinction and the IR PAH emission bands, however, is still missing. We present a new sample of hot stars that have both $2175 \AA$ absorption and IR PAH emission. We find significant shifts of the central wavelength of the UV absorption feature, up to $2350 \AA$, but predominantly in stars that also have IR PAH emission. These UV shifts depend on stellar temperature in a fashion that is similar to the shifts of the 6.2 and $7.7 \mu \mathrm{m}$ IR PAH bands, that is, the features are increasingly more redshifted as the stellar temperature decreases, but only below $\sim 15 \mathrm{kK}$. Above $15 \mathrm{kK}$ both UV and IR features retain their nominal values. Moreover, we find a suggestive correlation between the UV and IR shifts. We hypothesize that these similar dependences of both the UV and IR features on stellar temperature hint at a common origin of the two in PAH molecules and may establish the missing link between the UV and IR observations. We further suggest that the shifts depend on molecular size, and that the critical temperature of $\sim 15 \mathrm{kK}$ above which no shifts are observed is related to the onset of UV-driven hot-star winds and their associated shocks.
\end{abstract}

Key words: astrochemistry - dust, extinction - ISM: molecules

\section{Introduction}

The $2175 \AA$ ultraviolet (UV) extinction feature was discovered in 1965 by Stecher (1965) with sounding rocket observations. Since then, several satellite observatories have helped to characterize it toward many sightlines (Savage et al. 1985; Fitzpatrick \& Massa 1986, 1988, 1990, 2005, 2007; Cardelli et al. 1989; Valencic et al. 2004), yet its physical origin is still being debated. One suggested origin is absorption by polycyclic aromatic hydrocarbon (PAH) molecules, which are the most abundant organic molecules in nature and a key ingredient of the interstellar medium (ISM) (Tielens 2008). Indeed, theoretical molecular structure computations (Léger \& Puget 1984; Puget \& Léger 1989; Draine 2003; Zubko et al. 2004) as well as laboratory experiments (Steglich et al. 2010, 2011) indicate that PAHs absorb UV light and emit in broad mid-IR (MIR) bands. These works and other have ascribed the $2175 \AA$ feature to very small graphite grains (Stecher \& Donn 1965; Léger \& Puget 1984; Puget \& Léger 1989; Draine 2003; Zubko et al. 2004).

PAHs are organic molecules composed of $\mathrm{C}$ and $\mathrm{H}$ atoms, structured in the form of multiple aromatic rings with delocalized electrons. They contain up to $10 \%$ of the $\mathrm{C}$ content in our galaxy, and have thus been hypothesized to play a key role in the earliest forms of organic life in the universe (Ehrenfreund et al. 2006). PAHs are ubiquitous in the ISM, as revealed by their emission bands in the MIR around 3.3, 6.2, $7.7,8.6,11.2$, and $12.7 \mu \mathrm{m}$, originating in different vibrational modes (Allamandola et al. 1989). The typical temperature of ISM dust grains $(<100 \mathrm{~K})$ cannot account for efficient MIR emission, which is thus attributed to smaller species with low heat capacity, such as PAHs. The excitation energies of $\sim 10 \mathrm{eV}$ then imply typical molecule sizes of about 50 carbon atoms (Sellgren 1984). The PAH hypothesis is thus that the MIR PAH emission is excited by UV photons in the ISM, although perhaps not exclusively (Li \& Draine 2002; Smith et al. 2004).

MIR observations with the Infrared Space Observatory (ISO) and Spitzer space telescopes revealed the PAH emission band profiles and their relative intensities (Van Diedenhoven et al. 2004; Hony et al. 2001; Galliano et al. 2008; Mori et al. 2012). Observations showed a dependence of the central wavelength of the $6.2,7.7$, and $8.6 \mu \mathrm{m}$ bands on the illuminating stellar temperature (Sloan et al. 2007; Acke et al. 2010). The variation in band position has been attributed to varying size and charge of the PAH molecules (Pino et al. 2008; Ricca et al. 2012). The position of the UV extinction feature at $2175 \AA$, on the other hand, is rather uniform. This contrasts with the diversity of PAHs (Joblin et al. 1992) and ISM conditions, and thus challenges the PAH hypothesis. Nonetheless, a small number of cases have been reported where the UV extinction feature occurs at longer wavelengths (Greenstein 1981; Hecht et al. 1984; Buss et al. 1989).

Here, we use an analysis of a new sample of stars that have both UV and MIR spectra to demonstrate the missing connection between the UV absorption and MIR emission bands. The remainder of this paper is organized as follows. In Section 2 we describe the sample, in Section 3 we detail the measurements, in Section 4 we present the results, and in Section 5 we discuss the findings; we draw conclusions in Section 6.

\section{The Sample}

We assembled a sample of objects of spectral type A2 and earlier (hotter) that have spectra both from the IUE (UV), and 
Table 1

List of Stars, Spectral Properties, and UV Fitted Parameters

\begin{tabular}{|c|c|c|c|c|c|c|c|c|c|}
\hline $\begin{array}{l}\text { Object } \\
\#\end{array}$ & $\begin{array}{l}\text { Object } \\
\text { Name }\end{array}$ & $\begin{array}{l}\text { Spectral } \\
\text { Type }^{\mathrm{a}}\end{array}$ & $E(B-V)^{\mathrm{b}}$ & $\lambda_{0} \underset{(\AA)}{ \pm} \Delta \lambda_{0}$ & $\alpha \pm \Delta \alpha$ & $\tau \pm \Delta \tau$ & $w \underset{(\AA)}{ \pm} \pm_{\circ} \Delta w$ & $\chi^{2} /$ dof & $\begin{array}{l}\text { EW } \\
(\AA)\end{array}$ \\
\hline 1 & RR Tau & A0:IVe & 0.83 & $2307.2 \pm 24.0$ & $1.86 \pm 0.16$ & $0.69 \pm 0.42$ & $1060 \pm 210$ & 2.16 & 867 \\
\hline 2 & HD 95881 & A1/A2III/IV & 0.13 & $2400.2 \pm 12.8$ & $1.27 \pm 0.03$ & $0.30 \pm 0.08$ & $470 \pm 40$ & 4.73 & 352 \\
\hline 3 & HD 141569 & B9.5V & 0.09 & $2209.2 \pm 7.6$ & $0.24 \pm 0.01$ & $0.22 \pm 0.04$ & $310 \pm 20$ & 2.04 & 170 \\
\hline 4 & HD 31293 & $\mathrm{~A} 0 \mathrm{Ve}$ & 0.13 & $2261.1 \pm 13.9$ & $0.87 \pm 0.03$ & $0.20 \pm 0.08$ & $280 \pm 40$ & 4.34 & 151 \\
\hline 5 & HD 179218 & $\mathrm{~A} 0 \mathrm{Ve}$ & 0.11 & $2247.8 \pm 7.5$ & $0.30 \pm 0.01$ & $0.27 \pm 0.05$ & $370 \pm 30$ & 3.68 & 233 \\
\hline 6 & HD 97048 & A0Vep & 0.31 & $2218.1 \pm 2.9$ & $1.02 \pm 0.01$ & $0.42 \pm 0.03$ & $600 \pm 20$ & 0.81 & 483 \\
\hline 7 & HD 36917 & B9III/IV & 0.17 & $2253.2 \pm 8.5$ & $0.15 \pm 0.02$ & $0.28 \pm 0.06$ & $380 \pm 30$ & 2.63 & 255 \\
\hline 8 & HD 89353 & B9.5Ib-II & 0.37 & $2368.6 \pm 14.0$ & $4.98 \pm 0.02$ & $0.19 \pm 0.05$ & $480 \pm 50$ & 1.99 & 253 \\
\hline 9 & HD 97300 & B9V & 0.24 & $2203.1 \pm 4.4$ & $0.48 \pm 0.02$ & $0.42 \pm 0.05$ & $510 \pm 20$ & 1.10 & 405 \\
\hline 10 & HD 100546 & B9Vne & 0.48 & $2301.3 \pm 8.6$ & $-0.01 \pm 0.01$ & $0.18 \pm 0.04$ & $380 \pm 30$ & 2.37 & 170 \\
\hline 11 & HD 139636 & B8/9III & 0.29 & $2245.7 \pm 5.1$ & $0.68 \pm 0.03$ & $0.52 \pm 0.08$ & $650 \pm 40$ & 1.71 & 543 \\
\hline 12 & $\mathrm{BD}+30549$ & B8:p & 0.59 & $2177.0 \pm 2.9$ & $1.46 \pm 0.02$ & $0.60 \pm 0.05$ & $490 \pm 10$ & 1.59 & 530 \\
\hline 13 & HD 44179 & $\mathrm{~B} 9 \mathrm{Ib} / \mathrm{II}$ & 0.34 & $2364.6 \pm 15.3$ & $2.80 \pm 0.05$ & $0.39 \pm 0.11$ & $1110 \pm 100$ & 4.39 & 673 \\
\hline 14 & V699 Mon & B7IIne & 0.70 & $2241.7 \pm 4.8$ & $1.47 \pm 0.04$ & $0.75 \pm 0.08$ & $640 \pm 20$ & 2.71 & 762 \\
\hline 15 & HD 281159 & B5V & 0.83 & $2189.6 \pm 1.8$ & $1.94 \pm 0.02$ & $1.42 \pm 0.08$ & $540 \pm 10$ & 1.60 & 911 \\
\hline 16 & HD 124237 & B5/B6V & 0.48 & $2179.9 \pm 2.5$ & $1.31 \pm 0.02$ & $1.09 \pm 0.11$ & $380 \pm 20$ & 2.52 & 644 \\
\hline 17 & HD 147889 & B2III/IV & 1.05 & $2179.3 \pm 1.1$ & $0.89 \pm 0.02$ & $2.06 \pm 0.08$ & $510 \pm 10$ & 2.26 & 1024 \\
\hline 18 & $\mathrm{BD}+404124$ & $\mathrm{~B} 2 \mathrm{Ve}$ & 0.98 & $2216.6 \pm 3.0$ & $2.36 \pm 0.03$ & $3.80 \pm 1.21$ & $240 \pm 40$ & 4.49 & 849 \\
\hline 19 & HD 200775 & $\mathrm{~B} 2 \mathrm{Ve}$ & 0.61 & $2193.8 \pm 3.7$ & $1.63 \pm 0.02$ & $0.49 \pm 0.05$ & $560 \pm 20$ & 1.72 & 481 \\
\hline 20 & HD 37903 & B3IV & 0.31 & $2183.4 \pm 2.7$ & $0.82 \pm 0.01$ & $0.41 \pm 0.03$ & $440 \pm 10$ & 1.26 & 359 \\
\hline 21 & HD 36982 & $\mathrm{~B} 1.5 \mathrm{Vp}$ & 0.37 & $2201.9 \pm 3.5$ & $0.46 \pm 0.02$ & $0.45 \pm 0.04$ & $450 \pm 20$ & 1.36 & 403 \\
\hline 22 & cd-4911554 & B1Iae & 0.59 & $2154.7 \pm 4.6$ & $2.62 \pm 0.02$ & $0.42 \pm 0.06$ & $430 \pm 30$ & 2.42 & 353 \\
\hline 23 & SK-66 19 & $\mathrm{OB}$ & 0.43 & $2197.4 \pm 9.0$ & $2.07 \pm 0.02$ & $0.24 \pm 0.06$ & $330 \pm 30$ & 3.77 & 179 \\
\hline 24 & HD 37020 & B0V & 0.32 & $2215.2 \pm 10.4$ & $0.19 \pm 0.02$ & $0.17 \pm 0.04$ & $470 \pm 40$ & 2.42 & 183 \\
\hline 25 & cd-4211721 & BoIVe & 1.58 & $2160.3 \pm 6.2$ & $4.34 \pm 0.10$ & $1.71 \pm 0.38$ & $580 \pm 50$ & 1.88 & 1150 \\
\hline 26 & HD 37022 & O7Vp & 0.34 & $2215.1 \pm 4.7$ & $0.06 \pm 0.01$ & $0.24 \pm 0.03$ & $350 \pm 20$ & 1.05 & 191 \\
\hline 27 & HD 213985 & A0III & 0.16 & $2285.2 \pm 4.5$ & $1.20 \pm 0.03$ & $0.79 \pm 0.10$ & $820 \pm 40$ & 5.00 & 911 \\
\hline 28 & HD 105209 & A1V & 0.18 & $2268.0 \pm 15.1$ & $0.79 \pm 0.04$ & $0.22 \pm 0.07$ & $440 \pm 50$ & 2.22 & 212 \\
\hline 29 & HD 147009 & $\mathrm{~A} 0 \mathrm{~V}$ & 0.28 & $2233.0 \pm 3.3$ & $0.36 \pm 0.01$ & $0.58 \pm 0.05$ & $470 \pm 20$ & 1.89 & 489 \\
\hline 30 & HD 163181 & O9.5Ia/ab & 0.65 & $2170.6 \pm 2.3$ & $3.62 \pm 0.02$ & $1.43 \pm 0.16$ & $340 \pm 20$ & 3.28 & 692 \\
\hline 31 & HD 149914 & B9.5IV & 0.26 & $2212.0 \pm 4.2$ & $0.78 \pm 0.02$ & $0.49 \pm 0.06$ & $490 \pm 20$ & 1.74 & 478 \\
\hline 32 & HD 145554 & B9V & 0.20 & $2224.9 \pm 4.2$ & $0.29 \pm 0.01$ & $0.39 \pm 0.04$ & $410 \pm 20$ & 2.50 & 343 \\
\hline 33 & HD 58647 & B9IV & 0.14 & $2217.2 \pm 7.6$ & $0.50 \pm 0.02$ & $0.25 \pm 0.05$ & $320 \pm 30$ & 2.14 & 190 \\
\hline 34 & HD 147701 & B5III & 0.71 & $2176.2 \pm 3.2$ & $1.40 \pm 0.02$ & $0.83 \pm 0.06$ & $590 \pm 20$ & 2.39 & 726 \\
\hline 35 & HD 27396 & B4IV & 0.13 & $2197.9 \pm 5.7$ & $0.44 \pm 0.02$ & $0.30 \pm 0.04$ & $450 \pm 20$ & 2.13 & 286 \\
\hline 36 & HD 161573 & B4 & 0.17 & $2195.2 \pm 4.6$ & $0.37 \pm 0.01$ & $0.40 \pm 0.05$ & $400 \pm 20$ & 2.50 & 328 \\
\hline 37 & HD 38087 & B3II & 0.31 & $2192.5 \pm 1.9$ & $0.15 \pm 0.01$ & $0.54 \pm 0.03$ & $470 \pm 10$ & 1.21 & 458 \\
\hline 38 & HD 175156 & B3II & 0.33 & $2183.0 \pm 3.3$ & $1.92 \pm 0.02$ & $0.49 \pm 0.04$ & $440 \pm 20$ & 4.38 & 409 \\
\hline 39 & HD 141926 & B2nne & 0.74 & $2192.6 \pm 1.9$ & $2.16 \pm 0.02$ & $1.61 \pm 0.16$ & $370 \pm 20$ & 2.21 & 782 \\
\hline 40 & $\mathrm{BD}+622125$ & B5 & 0.73 & $2181.8 \pm 2.7$ & $3.09 \pm 0.03$ & $1.89 \pm 0.24$ & $350 \pm 20$ & 2.12 & 815 \\
\hline 41 & Hiltner188 & B1V & 0.54 & $2180.2 \pm 2.9$ & $3.03 \pm 0.03$ & $1.25 \pm 0.13$ & $390 \pm 20$ & 3.16 & 712 \\
\hline 42 & HD 154445 & $\mathrm{~B} 1 \mathrm{~V}$ & 0.38 & $2197.4 \pm 2.2$ & $0.65 \pm 0.02$ & $0.80 \pm 0.05$ & $450 \pm 10$ & 2.75 & 595 \\
\hline 43 & $\mathrm{BD}+56524$ & $\mathrm{~B} 1 \mathrm{Vn}$ & 0.6 & $2181.7 \pm 2.2$ & $2.34 \pm 0.02$ & $1.03 \pm 0.07$ & $390 \pm 10$ & 1.77 & 631 \\
\hline 44 & HD 239722 & B2IV & 0.78 & $2172.5 \pm 1.5$ & $3.40 \pm 0.02$ & $1.53 \pm 0.10$ & $430 \pm 10$ & 2.10 & 870 \\
\hline 45 & HD 23180 & B1III & 0.31 & $2136.1 \pm 4.9$ & $0.93 \pm 0.02$ & $0.50 \pm 0.07$ & $460 \pm 20$ & 3.24 & 440 \\
\hline 46 & HD 77581 & B0.5Ia & 0.73 & $2170.7 \pm 2.8$ & $3.39 \pm 0.03$ & $1.24 \pm 0.13$ & $400 \pm 20$ & 3.28 & 696 \\
\hline 47 & HD 239729 & B0V & 0.66 & $2181.5 \pm 2.7$ & $2.38 \pm 0.02$ & $0.73 \pm 0.05$ & $450 \pm 10$ & 2.45 & 554 \\
\hline 48 & CPD-592596 & B0V & 0.70 & $2195.5 \pm 2.5$ & $1.77 \pm 0.02$ & $0.81 \pm 0.06$ & $430 \pm 10$ & 3.42 & 570 \\
\hline 49 & HD 147165 & $09.5 \mathrm{~V}$ & 0.43 & $2184.5 \pm 2.8$ & $0.65 \pm 0.01$ & $0.64 \pm 0.05$ & $510 \pm 10$ & 3.37 & 540 \\
\hline 50 & $\mathrm{BD}+60594$ & O9V & 0.59 & $2182.8 \pm 2.8$ & $2.54 \pm 0.03$ & $1.23 \pm 0.10$ & $360 \pm 10$ & 3.25 & 642 \\
\hline 51 & HD 151804 & O8.5Iab(f)p & 0.36 & $2213.7 \pm 6.4$ & $1.43 \pm 0.02$ & $0.64 \pm 0.12$ & $220 \pm 20$ & 4.37 & 284 \\
\hline 52 & HD 149404 & O8.5Iab(f)p & 0.69 & $2203.6 \pm 2.4$ & $2.20 \pm 0.02$ & $1.39 \pm 0.13$ & $320 \pm 10$ & 4.04 & 616 \\
\hline 53 & HD 217086 & O7Vnn((f))z & 0.96 & $2200.7 \pm 1.7$ & $2.31 \pm 0.02$ & $1.65 \pm 0.10$ & $440 \pm 10$ & 2.01 & 855 \\
\hline 54 & HD 46150 & $\mathrm{O} 5 \mathrm{~V}((\mathrm{f})) \mathrm{z}$ & 0.46 & $2203.3 \pm 2.7$ & $1.39 \pm 0.02$ & $0.79 \pm 0.05$ & $380 \pm 10$ & 2.59 & 506 \\
\hline 55 & HD 93162 & O2.5If*/WN6 & 0.04 & $2207.5 \pm 3.4$ & $1.05 \pm 0.03$ & $0.79 \pm 0.07$ & $450 \pm 20$ & 4.47 & 594 \\
\hline 56 & HD 29647 & B8III & 1.02 & $2220.6 \pm 8.1$ & $4.16 \pm 0.05$ & $0.67 \pm 0.15$ & $1050 \pm 70$ & 2.85 & 1097 \\
\hline 57 & HD 132947 & B9V & 0.13 & $2216.9 \pm 8.5$ & $0.04 \pm 0.01$ & $0.24 \pm 0.05$ & $390 \pm 30$ & 2.25 & 224 \\
\hline 58 & HD 145631 & B9V & 0.20 & $2186.4 \pm 3.7$ & $0.32 \pm 0.01$ & $0.34 \pm 0.03$ & $410 \pm 10$ & 4.01 & 288 \\
\hline 59 & HD 23441 & $\mathrm{~A} 0 \mathrm{Vn}$ & 0.005 & $2239.2 \pm 11.6$ & $-0.36 \pm 0.01$ & $0.15 \pm 0.04$ & $350 \pm 40$ & 3.41 & 131 \\
\hline
\end{tabular}

Notes.

${ }^{a}$ Temperatures were determined from (Pecaut \& Mamajek (2013) according to spectral type.

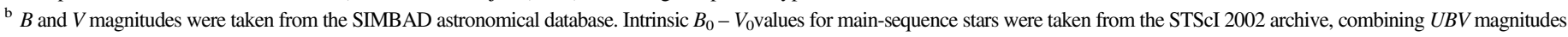

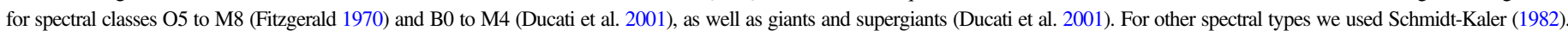


from either the Spitzer or ISO (MIR) telescopes. We did not impose any requirements on visible extinction; therefore, the present sample includes many stars with low color excess that would not be included in studies aimed at obtaining general extinction curves. We identified 27 stars that have both significant UV absorption (equivalent width, EW $\geqslant 100 \AA$ ) around $2175 \AA$ and significant PAH emission (EW $\geqslant 0.1 \mu \mathrm{m}$ in the $7.7 \mu \mathrm{m}$ band). Although not selected for it, most stars in the present sample happen to be pre-main-sequence stars that are shrouded in dust and gas, often in the form of a protoplanetary disk, which has been shown to provide favorable conditions for the growth of large PAH molecules (Berné et al. 2009). Besides its main attribute of including both UV and IR spectra, the present sample is unique in that it extends both to cooler stars (down to $8.25 \mathrm{kK}$ ) than those usually studied in the UV and to hotter stars (up to $37 \mathrm{kK}$ ) than those usually studied in the IR.

In order to test the UV-MIR connection and its relation to PAHs, we also constructed a control sample of 32 stars with UV absorption (EW $\geqslant 100 \AA$ ), but with no detectable MIR emission ( $\mathrm{EW} \leqslant 0.1 \mu \mathrm{m}$ at $7.7 \mu \mathrm{m}$ ), for comparison. A list of the present sample can be found in Table 1; objects 1-27 are those with both UV absorption and MIR PAH emission, while objects 28-59 are those with UV absorption but no detectable MIR emission. Spectral type and $B$ and $V$ magnitudes were taken from the SIMBAD astronomical database. ${ }^{5}$ Stellar temperatures $T_{\text {eff }}$ were determined from Pecaut \& Mamajek (2013) according to spectral type. Given the poor knowledge of the bolometric corrections, $T_{\mathrm{eff}}$ is uncertain by a few per cent (deJager \& Nieuwenhuijzen 1987), which is insignificant here. Intrinsic $B_{0}-V_{0}$ values for main-sequence stars were taken from the Space Telescope Science Institute (STScI) 2002 archive $^{6}$. For other spectral types we used the tables of Schmidt-Kaler (1982).

Despite the fact that the Hubble Space Telescope (HST) has improved UV capabilities compared to IUE, it had observed only about $10 \%$ of the number of UV-bright stars observed by IUE. For example, only two stars from our sample have adequate $H S T$ spectra, therefore we used the $I U E$ data. Moreover, in the two cases in which the HST and $I U E$ had observed the same objects the analyzed results were similar.

\section{Data Reduction and Analysis}

\section{1. $U V$}

The IUE spectra were extracted from the Mikulski Archive for Space Telescopes ${ }^{7}$, and comprise data obtained by three cameras. The short-wavelength camera (SWP) includes spectra from 1150 to $1978 \AA$, and the two long-wavelength cameras (LWP and LWR) include spectra from 1850 to $3347 \AA$, in both the large aperture and the small aperture. Observations that include at least one SWP spectrum and one LWP or LWR spectrum were selected. In the combined spectrum, the less noisy SWP is used up to $1970 \AA$, and LWP/LWR is used above $1970 \AA$. Where possible, we used the large IUE aperture since the small aperture can suffer from large flux variations during the observation. IUE bins flagged

\footnotetext{
5 http://simbad.u-strasbg.fr/simbad/

6 http://www.stsci.edu/ inr/intrins.html

7 https://archive.stsci.edu/iue/
}

for bad quality were ignored. Observations that include more than $20 \AA$ of sequentially flagged data, or more than $60 \AA$ in total in the wavelength range of interest, were omitted from the sample. In cases where several observations exist, we combined spectra bin-by-bin to obtain error-weighted $\left(1 / \sigma^{2}\right)$ means and the uncertainty of the mean $\left(1 / \sigma^{2}\right.$ added in quadrature). In cases where the large-aperture data did not comply with the above set of quality requirements, and smallaperture spectra exist, we completed the large-aperture spectrum with the small-aperture data, using a flux scaling based on the overlap regions.

The combined spectra were fitted between 1600 and $3200 \AA$. This range is similar to but larger than that of Fitzpatrick \& Massa (1986, 1988, 1990, 2005, 2007), Cardelli et al. (1989), and Valencic et al. (2004), and is much larger than the width of the feature for most stars. We fitted the IUE spectra directly and locally around the $2175 \AA$ feature using the stellar-atmospheric spectra of Castelli \& Kurucz (2004), modulated by a power law to represent the broadband extinction, the spectra were absorbed by a feature using a Drude profile. The measured flux spectrum $F_{\lambda}$ as a function of wavelength $\lambda$ then takes the following form:

$$
F_{\lambda}=F_{\mathrm{CK}} N\left(\frac{\lambda}{2000}\right)^{-\alpha} \exp \left(\frac{-A}{\pi} \frac{\lambda^{2}}{\left(\lambda^{2}-\lambda_{0}^{2}\right)^{2}+\lambda^{2} w^{2}}\right)
$$

where $F_{\mathrm{CK}}(\lambda)$ is the theoretical stellar spectrum (Castelli \& Kurucz 2004), $N$ is the normalization, and the power-law slope is $-\alpha$ (normalized at the arbitrary value of $2000 \AA$ ). In the exponent, $A$ represents the amplitude of the absorption feature, $w$ is the width, i.e., about the FWHM, and $\lambda_{0}$ is the central wavelength. The optical depth at the central wavelength is $\tau\left(\lambda_{0}\right)=A /\left(\pi \mathrm{w}^{2}\right)$. Equation (1) is fitted with the LevenbergMarquardt algorithm to the measured spectrum of each object by minimizing $\chi^{2}$. The best-fit values are consequentially obtained for the parameters $N, \alpha, \boldsymbol{A}, w$, and $\lambda_{0}$. For the stellar spectra, we used models of Castelli \& Kurucz (2004), based on the stellar spectral type. For each spectral type we fitted different metallicities and chose the metallicity that best fits the IUE spectrum. Most stars are fitted well. The few (3/62) stars with a reduced $\chi^{2} /$ dof $>5$ were eliminated from the sample. In Figure 1, we present a typical fit.

We compute the EW by integrating as follows:

$$
\mathrm{EW}=\frac{\int_{1600 \AA}^{3200 \AA}\left(F_{\lambda}^{0}-F_{\lambda}\right) d \lambda}{F_{\lambda}^{0}\left(\lambda_{0}\right)}
$$

where $F_{\lambda}^{0}$ is the unabsorbed continuum (Equation (1) with $A=0$ ). For the present study of PAH UV absorption, we retained only objects with $\mathrm{EW} \geqslant 100 \AA$. The results are listed in Table 1.

The table shows that regardless of the extinction $(E(B-V)$ and $\alpha$ ), we find shifted centroids, and varying optical depths and EWs. All uncertainties in the table reflect $2 \sigma(95.5 \%)$ confidence regions. These are estimated according to the standard procedure from the diagonal of the covariance matrix. The most important parameter for this paper is the centroid, and its fit is particularly robust, because it is independent of any of the other parameters. 


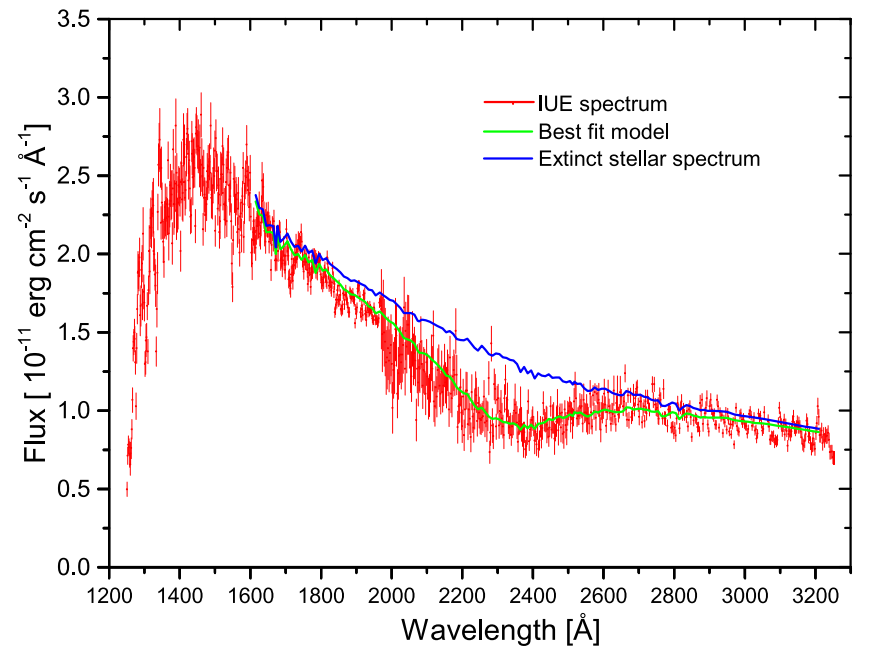

Figure 1. IUE spectrum and fitted model demonstrating significantly shifted absorption. Red data points are the IUE spectrum of HD 100546, the blue line is the stellar continuum from the model of Castelli \& Kurucz (2004) modified for extinction. The green line denotes the best-fitted model that includes also the Drude profile of the UV feature, whose central wavelength in HD 100546 is $2301 \AA$.

\section{2. $M I R$}

IR spectra were extracted for all the $59 \mathrm{UV}$-selected stars listed in Table 1 from IRSA, the NASA/IPAC Infrared Science Archive. ${ }^{8}$ We used data from the Spitzer/IRS instrument when available, and from ISO/SWS when not. For Spitzer, we combined the spectra from different slits between $5.13-7.6 \mu \mathrm{m}$, $7.33-8.66 \mu \mathrm{m}$, and $7.46-14.29 \mu \mathrm{m}$ to obtain a continuous spectrum between 5.13 and $14.29 \mu \mathrm{m}$. For $I S O$, the data extracted from IRSA are already combined (Sloan et al. 2003). The brightest PAH emission bands are around 3.3, 6.2, 7.7, 8.6, and $11.2 \mu \mathrm{m}$. We measured their individual fluxes by fitting Gaussians over the local continuum. We also tried Lorentzian profiles, as well as simple spline functions (Galliano et al. 2008). It has been shown that the resulting flux is only slightly affected by the chosen profile, while it may depend somewhat on the determination of the continuum (Uchida et al. 2000). The central wavelength is much less affected by the choice of profile function. Since all of our data were fitted with the same method, the trends and conclusions do not change with such small systematic uncertainties. A sample IR spectrum is shown in Figure 2.

About half $(27 / 59)$ of the sources in our sample have a measurable MIR emission band around $7.7 \mu \mathrm{m}$ with an $\mathrm{EW} \geqslant 0.1 \mu \mathrm{m}$. This half of the sample is referred to here as stars with MIR PAH emission, while the other stars are referred to as being without MIR PAH emission. For stars with MIR PAH emission, we fitted the flux and central wavelength of the 6.2 and $7.7 \mu \mathrm{m}$ bands. The results are listed in Table 2.

\section{Results}

We find no particular pattern or correlation with feature strength (EW) either in the UV or in the MIR. We thus focus on the central wavelength $\lambda_{0}$ (i.e., the position) of the features, which we show below to be highly revealing.

Figure 3 shows the position of the UV absorption feature $\lambda_{0}$ as a function of $T_{\text {eff }}$. The longest wavelengths are observed in the relatively cool stars with $8.25 \mathrm{kK}<T_{\text {eff }}<14.5 \mathrm{kK}$. In

\footnotetext{
http://irsa.ipac.caltech.edu/frontpage/
}

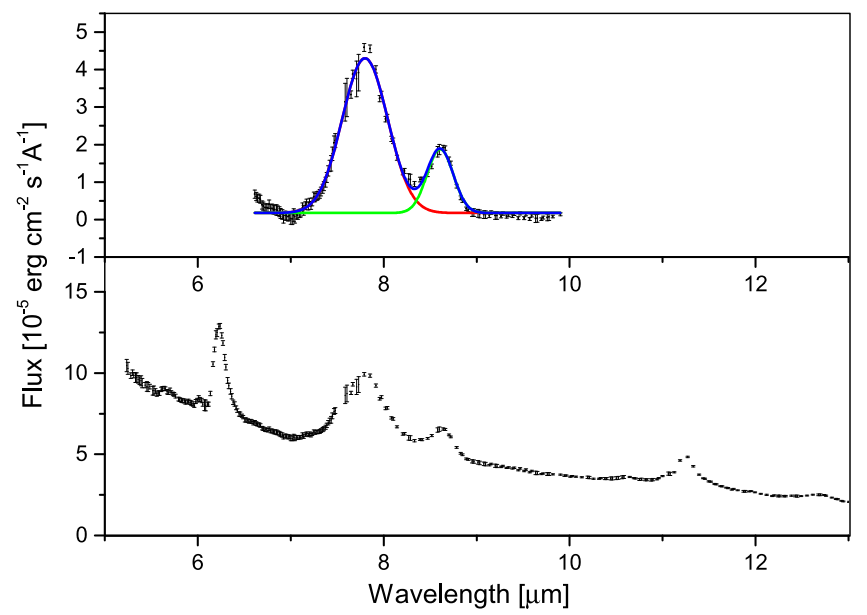

Figure 2. Spitzer spectrum and fitted model demonstrating shifted emission. Top panel: a two-Gaussian fit of the redshifted 7.7 and $8.6 \mu \mathrm{m}$ emission bands of RR Tau. A local linear continuum has been subtracted out. Bottom panel: the full Spitzer spectrum of RR Tau in which multiple PAH bands are clearly seen.

these objects, the central UV wavelength can shift up to $2400 \AA$, while the hotter stars with $T_{\text {eff }}>14.5 \mathrm{kK}$ approximately follow a nominal value of $2195 \pm 40 \AA$. Furthermore, the most extreme (long) UV wavelengths are observed in those stars that also have PAH emission bands in the MIR. A Kolmogorov-Smirnov (KS) test on the cool stars from the main and control samples gives a probability of only 0.037 that they are drawn from the same underlying sample.

Figure 4 presents the central wavelengths of the MIR PAH emission bands. For the 6.2 and $7.7 \mu \mathrm{m}$ emission bands, the longer MIR central wavelengths appear preferentially in the cooler host stars, while hotter host stars feature nominal values of $6.23 \pm 0.015 \mu \mathrm{m}$ and $7.66 \pm 0.05 \mu \mathrm{m}$. We find no significant shifts in the 8.6, 11.2, and $12.7 \mu \mathrm{m}$ bands, in line with the results of Van Diedenhoven et al. (2004).

Figure 5 presents the direct correspondence between the position of the UV absorption feature and that of the $7.7 \mu \mathrm{m}$ emission band for stars with $T_{\text {eff }}<14.5 \mathrm{kK}$. A reasonably good correlation is found between the two shifts, with Pearson's $R=0.57$ and a chance probability of 0.021 . The correlation improves to $R=0.77$ and a chance probability of $8.1 \times 10^{-4}$ if the significantly outlying star on the upper left side (HD 141569) is ignored. The EW of HD 141569 (170 ̊) is the second lowest in our sample.

\section{Discussion}

\subsection{UV Shifts}

Previous UV samples (Fitzpatrick \& Massa 1986, 1988, 1990, 2005, 2007; Cardelli et al. 1989; Valencic et al. 2004) showed that the central wavelength $\lambda_{0}$ was almost constant, with $\lambda_{0}=2178.5 \pm 9.1 \AA$ (Fitzpatrick \& Massa 2007), and with a maximum range of 2130-2222 $\AA$ (Valencic et al. 2004). The present sample, however, has a much broader and also shifted distribution of $\lambda_{0}$. This is particularly the case for the stars that have MIR PAH emission bands, and less so for those that do not. Stars with and without MIR PAH emission, respectively, have weighted means of 2192.3 and $2189.5 \AA$. The standard deviation of $63.9 \AA$ is large for stars with MIR PAH emission, and less $(24.2 \AA)$ for those without it. The present sample has more shifted UV features than previous 
Table 2

List of Stars with MIR PAH Bands, and Fitted Parameters

\begin{tabular}{|c|c|c|c|c|c|}
\hline \# & Object Name & Telescope & Spectral Type & $\begin{array}{c}6.2 \mu \mathrm{m} \text { Band Center } \\
\lambda_{0} \pm \Delta \lambda_{0}(\mu \mathrm{m})\end{array}$ & $\begin{array}{c}\text { 7.7 } \mu \mathrm{m} \text { Band Center } \\
\lambda_{0} \pm \Delta \lambda_{0}(\mu \mathrm{m})\end{array}$ \\
\hline 1 & RR Tau & Spitzer & A0:IVe & $6.239 \pm 0.0021$ & $7.798 \pm 0.0036$ \\
\hline 2 & HD 95881 & Spitzer & A1/A2III/IV & $6.273 \pm 0.0047$ & $7.932 \pm 0.009$ \\
\hline 3 & HD 141569 & Spitzer & $\mathrm{B} 9.5 \mathrm{~V}$ & $6.267 \pm 0.0069$ & $7.909 \pm 0.0073$ \\
\hline 4 & HD 31293 & ISO & $\mathrm{A} 0 \mathrm{Ve}$ & $6.262 \pm 0.0039$ & $7.789 \pm 0.0124$ \\
\hline 5 & HD 179218 & $I S O$ & $\mathrm{~A} 0 \mathrm{Ve}$ & $6.276 \pm 0.0011$ & $7.8 \pm 0.0012$ \\
\hline 6 & HD 97048 & Spitzer & A0Vep & $6.232 \pm 0.0017$ & $7.781 \pm 0.0035$ \\
\hline 7 & HD 36917 & Spitzer & B9III/IV & $6.23 \pm 0.0036$ & $7.711 \pm 0.0081$ \\
\hline 8 & HD 89353 & ISO & B9.5Ib-II & $6.265 \pm 0.0006$ & $7.823 \pm 0.0017$ \\
\hline 9 & HD 97300 & Spitzer & B9V & $6.235 \pm 0.0027$ & $7.715 \pm 0.0042$ \\
\hline 10 & HD 100546 & ISO & B9Vne & $6.25 \pm 0.0005$ & $7.901 \pm 0.0007$ \\
\hline 11 & HD 139636 & Spitzer & B8/9III & $6.257 \pm 0.0052$ & $7.752 \pm 0.016$ \\
\hline 12 & $\mathrm{BD}+30549$ & Spitzer & B8:p & $6.221 \pm 0.0042$ & $7.69 \pm 0.0056$ \\
\hline 13 & HD 44179 & ISO & $\mathrm{B} 9 \mathrm{Ib} / \mathrm{II}$ & $6.268 \pm 0.0005$ & $7.827 \pm 0.0004$ \\
\hline 14 & V699 Mon & Spitzer & B7IIne & $6.232 \pm 0.0035$ & $7.687 \pm 0.0046$ \\
\hline 15 & HD 281159 & Spitzer & $\mathrm{B} 5 \mathrm{~V}$ & $6.219 \pm 0.0045$ & $7.646 \pm 0.0173$ \\
\hline 16 & HD 124237 & Spitzer & B5/B6V & $6.225 \pm 0.0053$ & $7.719 \pm 0.0109$ \\
\hline 17 & HD 147889 & Spitzer & B2III/IV & $6.221 \pm 0.0066$ & $7.651 \pm 0.0089$ \\
\hline 18 & $\mathrm{BD}+404124$ & Spitzer & $\mathrm{B} 2 \mathrm{Ve}$ & $6.221 \pm 0.0007$ & $7.657 \pm 0.0017$ \\
\hline 19 & HD 200775 & Spitzer & $\mathrm{B} 2 \mathrm{Ve}$ & $6.232 \pm 0.0035$ & $7.612 \pm 0.0061$ \\
\hline 20 & HD 37903 & Spitzer & B3IV & $6.236 \pm 0.0031$ & $7.659 \pm 0.007$ \\
\hline 21 & HD 36982 & Spitzer & $\mathrm{B} 1.5 \mathrm{Vp}$ & $6.234 \pm 0.0037$ & $7.659 \pm 0.0046$ \\
\hline 22 & cd-4911554 & ISO & B1Iae & $6.228 \pm 0.004$ & $7.801 \pm 0.0027$ \\
\hline 23 & SK-66 19 & Spitzer & OB & $6.231 \pm 0.0084$ & $7.7 \pm 0.0296$ \\
\hline 24 & HD 37020 & Spitzer & $\mathrm{B} 0 \mathrm{~V}$ & $6.229 \pm 0.0026$ & $7.572 \pm 0.0241$ \\
\hline 25 & cd-4211721 & $I S O$ & BOIVe & $6.227 \pm 0.0008$ & $7.667 \pm 0.0012$ \\
\hline 26 & HD 37022 & Spitzer & O7Vp & $6.229 \pm 0.0027$ & $7.64 \pm 0.0055$ \\
\hline 27 & HD 213985 & Spitzer & A0III & $\ldots$ & $7.83 \pm 0.0017$ \\
\hline
\end{tabular}

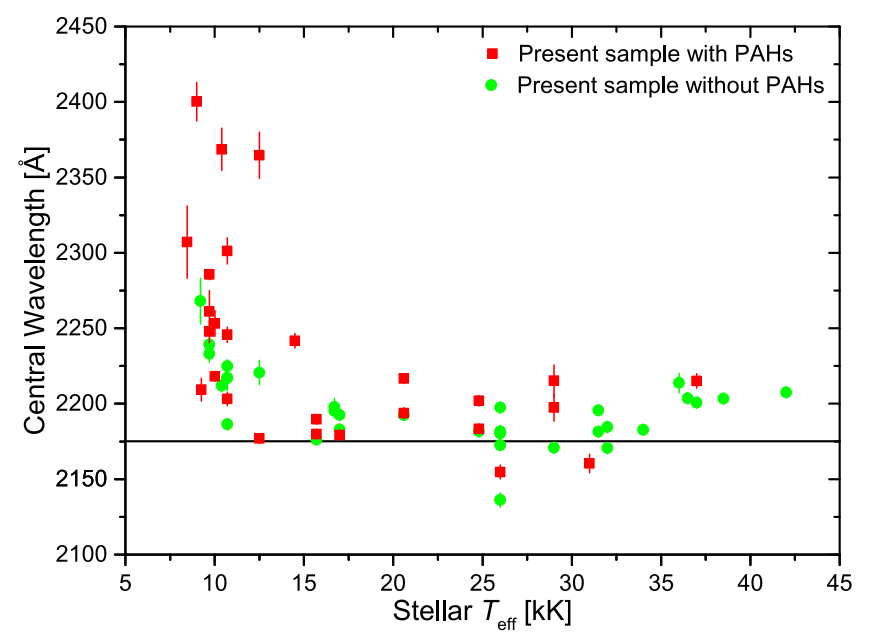

Figure 3. Directly measured central wavelength of the broad UV interstellar absorption feature along lines of sight to stars with different effective temperatures. All stars with $T_{\text {eff }}>14.5 \mathrm{kK}$ lie within $2195 \pm 40 \AA$, which is consistent with the nominal value of $2175 \AA$ (horizontal line). In the cooler stars with $8 \mathrm{kK}<T_{\text {eff }}<14.5 \mathrm{kK}$, the central UV wavelength can shift up to $2400 \AA$.

samples because it was selected differently. We did not impose any requirements on visible extinction, nor assume a standard extinction curve. In fact, our sample includes many stars with low color excess that, fitted locally, show a strong UV feature. In addition, our method allows the stellar metallicity to deviate from solar and thus improve the fit to the observed spectrum.

A few early claims in the literature of shifts of the UV feature toward longer wavelength attributed it to carbon-rich

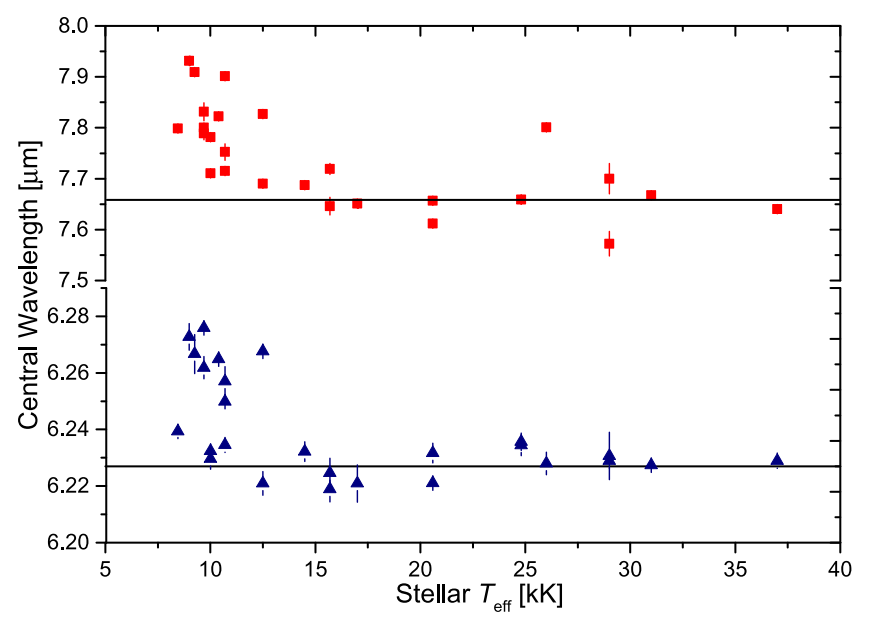

Figure 4. Central wavelength of the 7.7 and $6.2 \mu \mathrm{m}$ PAH bands for stars from the present sample with different effective temperatures. The central wavelength of the MIR PAH bands in hot stars is consistent with the nominal positions at $6.23 \pm 0.015 \mu \mathrm{m}$ and at $7.66 \pm 0.05 \mu \mathrm{m}$ (horizontal lines). As the temperature drops below $T_{\text {eff }}<14.5 \mathrm{kK}$, the central wavelength gradually increases with decreasing temperature.

environments around the stars (Greenstein 1981; Hecht et al. 1984; Buss et al. 1989). We analyzed the spectra of all of these sources. The objects HD 89353 and HD 213985, discussed by Buss et al. (1989), are included in our sample, and both show strong MIR PAH emission (see Table 1). On the other hand, the planetary nebula Abell 30 (Greenstein 1981) and R Y Sgr and R CrB (Hecht et al. 1984) do not have significant UV absorption, and as such do not qualify for 


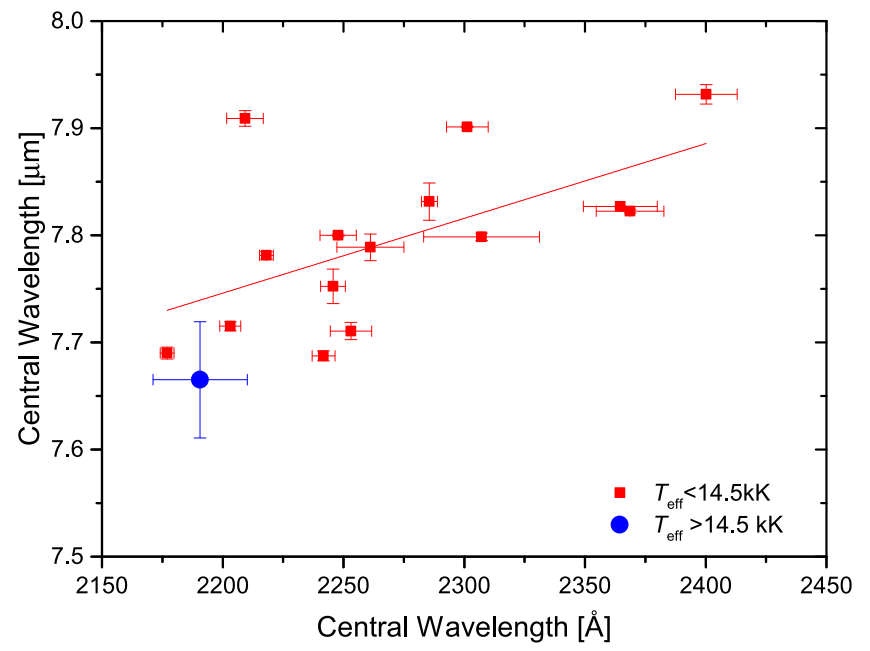

Figure 5. Anomalously long central wavelengths of the $2175 \AA$ UV absorption feature and the $7.7 \mu \mathrm{m}$ PAH emission band. The lower temperature $\left(T_{\text {eff }}<14.5 \mathrm{kK}\right)$ part of the present sample (red squares) shows redshifts of both UV and MIR features from their respective nominal regimes around $2195 \AA$ and $7.66 \mu \mathrm{m}$ (blue data point and error bars) where most stars, including all of the hotter ones, reside.

inclusion in the present sample, nor can they represent regions of the ISM where UV absorption can be studied.

\subsection{MIR Shifts}

The dependence of the MIR PAH shifts on $T_{\text {eff }}$ in Figure 4 corroborates previously published results, as can be seen in Figure 6 , where the position of the $7.7 \mu \mathrm{m}$ band as a function of $T_{\text {eff }}$ continues to increase down to $4 \mathrm{kK}$ (data from Sloan et al. 2007; Acke et al. 2010). The present sample extends the trend to higher temperatures and demonstrates that the central wavelength tends to the nominal value of $\sim 7.66 \mu \mathrm{m}$ above $15 \mathrm{kK}$. This result is no different to the findings of Peeters et al. (2002) that the MIR PAH central wavelengths change with stellar type (and hence temperature). Note that stellar temperature cannot be the only parameter governing the MIR shift, because the $7.7 \mu \mathrm{m}$ feature around planetary nebulae $(\mathrm{PNe})$, where $T_{\text {eff }}$ can reach $100 \mathrm{kK}$, is also shifted to $7.9 \mu \mathrm{m}$ (Joblin et al. 2008).

The limitation of the statistical correlation notwithstanding, the important result here is that stars whose UV absorption is redshifted also have redshifted MIR PAH bands, both beyond the high- $T_{\text {eff }}$ average (blue data point in Figure 5), suggesting that PAHs are responsible for at least the shifted UV absorption.

The correlation presented in Figure 5 shows that many IRshifted sources in the sample appear specifically at $7.9 \mu \mathrm{m}$. This feature was identified in protoplanetary disks (Boersma et al. 2009) and UV-bright PNe with large PAH molecules, while here it is found in the weakest UV (low $T_{\text {eff }}$ ) sources. The scatter in the correlation of Figure 5 is likely due to more complicated molecular astrochemistry and the varying circumstellar environments, but the upshot is that stars that feature redshifted UV absorption also feature redshifted MIR emission bands. This result, in conjunction with the same pattern of the UV absorption and MIR emission shifting with decreasing stellar temperature, suggests that the UV absorption feature around $2200 \AA$ is a result of the same molecules that emit the MIR bands, and which are believed to be PAHs.

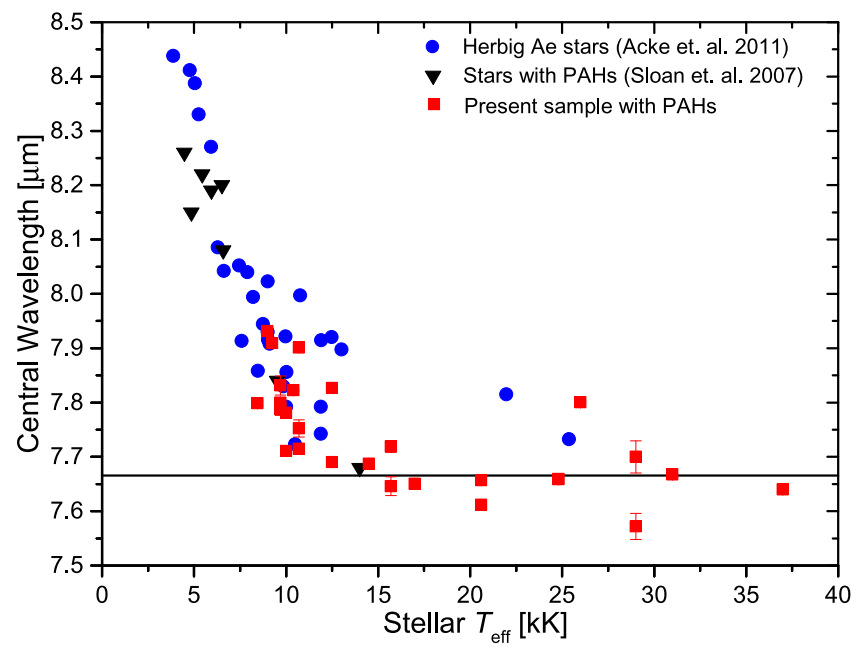

Figure 6. Central wavelength of the $7.7 \mu \mathrm{m}$ MIR PAH band for stars with different effective temperatures. The dependence of the central wavelength on stellar temperature from the literature (Sloan et al. 2007; Acke et al. 2010) is extended to higher temperatures of up to $37 \mathrm{kK}$ using the present UV-selected sample of hot stars.

\subsection{What Drives the Shifts in UV and MIR Wavelength?}

Longer UV and MIR wavelengths indicate larger PAH molecules with more than 50 carbon atoms (Bauschlicher et al. 2010; Steglich et al. 2010, 2011; Cami 2011; Ricca et al. 2012), as can also be seen by comparing, e.g., cross sections of coronene $\left(\mathrm{C}_{24} \mathrm{H}_{12}\right)$ versus circumcoronene $\left(\mathrm{C}_{54} \mathrm{H}_{18}\right)$, or ovalene $\left(\mathrm{C}_{32} \mathrm{H}_{14}\right)$ versus circumovalene $\left(\mathrm{C}_{66} \mathrm{H}_{20}\right)$ (Malloci et al. 2007). Redshifted MIR bands tend to arise from larger molecules as well, but ionization (Malloci et al. 2007), nitrogen atoms in the aromatic rings (Hudgins et al. 2005), and aliphatic compounds (Sloan et al. 2007) can also shift their central wavelength.

Although PAH UV shifts have not been studied systematically, and although several effects can cause MIR shifts, the present correlation between the two and the similar dependence on $T_{\text {eff }}$ lend support to the size playing a dominant role. If this interpretation is correct, larger molecules are present around the cooler stars, but not around the hot ones, with $T_{\text {eff }} \sim 15 \mathrm{kK}$ being the dividing temperature.

Note that Seok et al. (2017), who find similar MIR trends with stellar temperature, reach the opposite conclusion about the size, namely that redshifts indicate smaller molecules are present around the cool stars. This shows that spectroscopic signatures of molecular sizes in the MIR and in the UV are still poorly understood and need further study.

\subsection{What is Special about $15 \mathrm{kK}$ ?}

Both the UV and MIR features start being significantly shifted only below $\sim 15 \mathrm{kK}$. This is the critical temperature for stellar winds driven by UV pressure (Lamers et al. 1995; Prinja \& Massa 1998; Kudritzki \& Puls 2000). Although one might expect the UV flux from the hot stars to destroy the smaller molecules, this is not what is observed here. Another possible effect of the hot-star winds may have to do with the shocks and the hot (megakelvin) X-ray gas that they produce. If shock heating destroys preferentially the large molecules, it could explain why no UV and MIR shifts in wavelength are observed in these hot stars, where the nominal wavelengths of $2175 \AA$ and $7.66 \mu \mathrm{m}$ may represent the normal ISM composition of dust grains and normal-size PAHs with $\lesssim 50$ carbon atoms 
(Sellgren 1984; Tielens 2008). This speculation remains to be investigated in future studies.

\subsection{Is the Phenomenon Circumstellar or Interstellar?}

One may wonder whether the absorbing and emitting molecules are associated with the background stars or are typical of mean ISM properties. The similar behavior of the shifts in UV and IR wavelengths, as well as the dependence on stellar temperature, strongly suggests that these features are associated with the host stars. Moreover, we find no correlation between the measured UV EW and the Galactic latitude (a correlation coefficient of determination $R^{2}=0.14$ ), or the EW and the stellar distance $\left(R^{2}=0.09\right)$. This is in fact expected for the present sample, which consists mostly of nearby stars and for which the measured UV and MIR properties are not associated with mean ISM values along the random lines of sight.

One can estimate mean ISM UV absorption and IR emission based on the expected absorbed and emitted fluxes in the ISM (Siebenmorgen et al. 2014). The mean H number density $n_{\mathrm{H}}$ in the ISM along a random line of sight can be expected to range between 0.1 and $1.0 \mathrm{~cm}^{-3}$. Assuming a solar $\mathrm{C}$ abundance of $\mathrm{C} / \mathrm{H}=2.4 \times 10^{-4}$, and that $10 \%$ of the $\mathrm{C}$ is in PAHs, the number density of $\mathrm{C}$ atoms in PAHs is $n_{\mathrm{PAH}_{C}}=2.4 \times$ $10^{-5} n_{\mathrm{H}}$. The column density toward a star is then the mean density times the distance to the star. The most distant stars in our sample are $\sim 2 \mathrm{kpc}\left(6.2 \times 10^{21} \mathrm{~cm}\right)$ away, which corresponds to column densities of $N_{\mathrm{H}}=6.2 \times 10^{21} n_{\mathrm{H}} \mathrm{cm}^{-2}$ and $n_{\mathrm{PAH}_{C}}=1.5 \times 10^{17} n_{\mathrm{H}}$.

The UV PAH absorption cross section at the center of the feature $\left(\lambda_{0}\right)$ is $\sigma=10^{-17} \mathrm{~cm}^{2}$ per PAH C atom (Siebenmorgen et al. 2014). Hence, the mean ISM optical depth is expected to be $\tau\left(\lambda_{0}\right)=\sigma n_{\mathrm{PAH}_{C}} d$, where $d$ is the distance to the star. If $d_{\mathrm{pc}}$ is the distance measured in parsecs, one can plug in the numbers and write simply

$$
\tau\left(\lambda_{0}\right)=7.4 \times 10^{-4} n_{\mathrm{PAH}_{C}} d_{\mathrm{pc}} .
$$

We were able to find distances to 51 out of the 59 stars in the sample, most of which are closer than $500 \mathrm{pc}$ and therefore are not expected to have much ISM absorption. In Figure 7, we compare the analytic estimate with the optical depth $\tau\left(\lambda_{0}\right)=A /\left(\pi w^{2}\right)$ measured from the fitted spectra. It is demonstrated that all of the current measurements lie much above the line for $n_{\mathrm{H}}=0.1 \mathrm{~cm}^{-3}$, and all but two stars lie above the line for $n_{\mathrm{H}}=1 \mathrm{~cm}^{-3}$.

In the IR, the intensity of the $7.7 \mu \mathrm{m}$ band per $\mathrm{H}$ column density is $\nu I_{\nu} / N_{\mathrm{H}}=5.6 \times 10^{-26} \mathrm{erg} \mathrm{s}^{-1} \mathrm{~cm}^{-2} \mathrm{sr}^{-1}$ (Siebenmorgen et al. 2014). Using $N_{\mathrm{H}}$ from above, the intensity up to $2 \mathrm{kpc}$ would be $\nu I_{\nu}=3.1 \times 10^{-4} n_{\mathrm{H}}$ $\mathrm{erg} \mathrm{s}^{-1} \mathrm{~cm}^{-2} \mathrm{sr}^{-1}$. The Spitzer/IRS spectra we use are reduced from a $20 \operatorname{arcsec}^{2}$ extraction $\left(4.7 \times 10^{-10} \mathrm{sr}\right)$ on the sky. Thus, the expected mean ISM flux in the $7.7 \mu \mathrm{m}$ band is

$$
\nu F_{\nu}=1.5 \times 10^{-13} n_{\mathrm{H}} \mathrm{erg} \mathrm{s}^{-1} \mathrm{~cm}^{-2} .
$$

The integrated flux of the feature is approximately $F_{\nu} \Delta \nu=$ $\nu F_{\nu}(\Delta \nu / \nu)=\nu F_{\nu}(\Delta \lambda / \lambda)$. The measured width (FWHM) of the $7.7 \mu \mathrm{m}$ feature is on average $\Delta \lambda=0.6 \mu \mathrm{m}$, or $\Delta \lambda / \lambda=0.078$, thus the expected flux of the feature due to the ISM emission is $F_{\nu} \Delta \nu=1.2 \times 10^{-14} n_{\mathrm{H}} \mathrm{erg} \mathrm{s}^{-1} \mathrm{~cm}^{-2}$, even for a distant target $2 \mathrm{kpc}$ away. For the range of $n_{\mathrm{H}}$ between 0.1 and $1.0 \mathrm{~cm}^{-3}$, we thus obtain $F_{\nu} \Delta \nu \approx 10^{-15}-10^{-14} \mathrm{erg} \mathrm{s}^{-1} \mathrm{~cm}^{-2}$, which is far

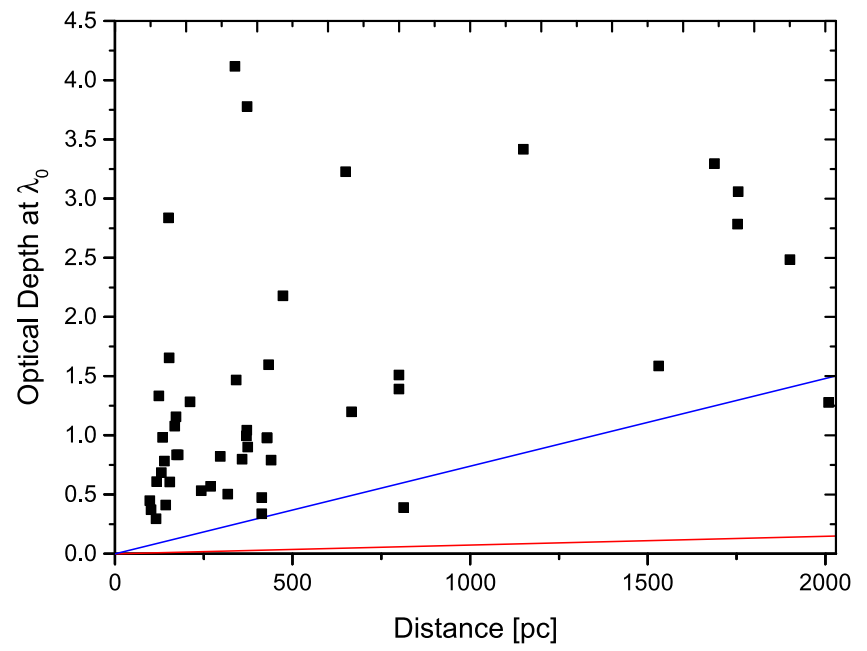

Figure 7. UV optical depth as a function of distance. The optical depth at the center of the UV feature is plotted as a function of the distance for 49 out of the 59 stars in the present sample. Three stars fall outside the plot (one with $\tau>7$ and two with $d>2.5 \mathrm{kpc}$ ), and for seven we could not find the distance. An analytical (linear) estimate for $\tau$ is plotted for $n_{\mathrm{H}}=0.1 \mathrm{~cm}^{-3}$ (lower line) and for $1.0 \mathrm{~cm}^{-3}$ (upper line). The mean ISM optical depth is expected to fall between these two lines, demonstrating that the UV absorption measured in the sample is not commensurate with ISM absorption and can be mostly ascribed to the stellar environment.

weaker than the flux range of $3.5 \times 10^{-13}$ to $2 \times 10^{-9}$ $\mathrm{erg} \mathrm{s}^{-1} \mathrm{~cm}^{-2}$ measured in our sample. We conclude therefore that the UV and IR measurements toward stars in the present sample can mostly be associated with the stellar environment and not with the random, mean ISM properties of the individual lines of sight.

\section{Summary and Conclusions}

Our results can be summarized as follows.

1. We find significant shifts of the UV extinction feature around $2200 \AA$.

2. These shifts become more appreciable as the temperature of the background star decreases below $15 \mathrm{kK}$, where hotstar winds cease to exist.

3. Exactly the same trend of shifts with stellar temperature occurs also in MIR PAH bands.

4. We find the shifts in the UV to correlate with those in the MIR, suggesting that they both originate in the physical properties of PAH molecules.

5. One possible reason for the shifts could be molecular size.

These results open new possibilities to diagnose specific molecular species in the ISM, which cannot be done in the MIR alone, but could be achieved with more UV laboratory and computational experiments. These could test whether the shifts are due to molecular size. While the present results associate large PAH molecules preferentially with windless, planetforming stars, the astrophysical setting for the formation and survival of these organic molecules, and their possible connection to the origin of life, remains to be investigated. Moreover, our findings will allow one to predict the presence of PAHs in the ISM and the positions of their MIR bands from the UV spectra, and vice versa. Furthermore, the observed variation in the $2175 \AA$ feature may be the long sought-after electronic signature of PAHs in the UV. 
Work at the Technion was supported by the I-CORE program of the Planning and Budgeting Committee (grants $1937 / 12$ \& 1829/12). A.B. acknowledges programming support from Uria Peretz. E.B. received funding from the European Union's Horizon 2020 research and innovation programme under the Marie Sklodowska-Curie grant agreement no. 655324.

\section{References}

Acke, B., Bouwman, J., Juhász, A., et al. 2010, ApJ, 718, 558

Allamandola, L. J., Tielens, A. G. G. M., \& Barker, J. R. 1989, ApJS, 71, 733A Bauschlicher, C. W., Jr., Boersma, C., Ricca, A., et al. 2010, ApJS, 189, 341

Berné, C., et al. 2009, A\&A, 495, 827

Boersma, C., et al. 2009, A\&A, 502, 175

Buss, R. H., Jr., Snow, T. P., Jr., \& Lamers, H. J. G. L. M. 1989, ApJ, 347, 977

Cami, J. 2011, EAS, 46, 117

Cardelli, J. A., Clayton, G. C., \& Mathis, J. S. 1989, ApJ, 345, 245

Castelli, F., \& Kurucz, R. L. 2004, arXiv:astro-ph/0405087

deJager, C., \& Nieuwenhuijzen, H. 1987, A\&A, 177, 217

Draine, B. T. 2003, ARA\&A, 41, 241

Ducati, J. R., Bevilacqua, C. M., Rembold, S. B., Ribeiro, D., et al. 2001, ApJ, 558, 309

Ehrenfreund, P., et al. 2006, AsBio, 6, 490

Fitzgerald, M. P. 1970, A\&A, 4, 234

Fitzpatrick, E. L., \& Massa, D. 1986, ApJ, 307, 286

Fitzpatrick, E. L., \& Massa, D. 1988, ApJ, 328, 734

Fitzpatrick, E. L., \& Massa, D. 1990, ApJ, 72, 163

Fitzpatrick, E. L., \& Massa, D. 2005, ApJ, 130, 1127

Fitzpatrick, E. L., \& Massa, D. 2007, ApJ, 663, 320

Galliano, F., Madden, S. C., Tielens, A. G. G. M., Peeters, E., \& Jones, A. P. 2008, ApJ, 679, 310

Greenstein, J. L. 1981, ApJ, 245, 124

Hecht, J. H., Holm, A. V., Donn, B., \& Wu, C.-C. 1984, ApJ, 280, 228

Hony, S., Van Kerckhoven, C., Peeters, E., et al. 2001, A\&A, 370, 1030
Hudgins, D. M., Bauschlicher, C. W., \& Allamandola, L. J. 2005, ApJ, 632, 316

Joblin, C., Léger, A., \& Martin, P. 1992, ApJL, 393, L79

Joblin, C., Szczerba, R., Berné, O., \& Szyszka, C. 2008, A\&A, 490, 189

Kudritzki, R.-P., \& Puls, J. 2000, ARA\&A, 3, 613

Lamers, H. J. G. L. M., Snow, T. P., \& Lindholm, D. M. 1995, ApJ, 455, 269

Léger, A., \& Puget, J. 1984, A\&A, 137, L5

Li, A., \& Draine, B. T. 2002, ApJ, 572, 232

Malloci, G., Joblin, C., \& Mulas, G. 2007, CP, 332, 353

Mori, T. I., Sakon, I., Onaka, T., et al. 2012, ApJ, 744, 68

Pecaut, \& Mamajek 2013, ApJS, 208, 9

Peeters, E., Hony, S., Van Kerckhoven, C., et al. 2002, A\&A, 390, 1089

Pino, T., Dartois, E., Cao, A.-T., et al. 2008, A\&A, 490, 665

Prinja, R. K., \& Massa, D. L. 1998, ASPC, 131, 218

Puget, J. L., \& Léger, A. 1989, ARA\&A, 27, 161

Ricca, A., Bauschlicher, C. W., Jr., Boersma, C., et al. 2012, ApJ, 754, 75

Savage, B. D., Massa, D., Meade, M., \& Wesselius, P. R. 1985, ApJ, 59, 397

Schmidt-Kaler 1982, http://obswww.unige.ch/gcpd/mk01bv.html

Sellgren, K. 1984, ApJ, 277, 623

Seok, J. Y., \& Li, A. 2017, ApJ, 835, 291

Siebenmorgen, R., Voshchinnikov, N. V., \& Bagnulo, S. 2014, A\&A, $561 \mathrm{~A}, 82$

Sloan, G. C., Jura, M., Duley, W. W., et al. 2007, ApJ, 664, 1144

Sloan, G. C., Kraemer, K. E., Price, S. D., \& Shipman, R. F. 2003, ApJS, 147,379

Smith, T. L., Clayton, G. C., \& Valencic, L. 2004, AJ, 128, 357

Stecher, T. P. 1965, ApJ, 142, 1683

Stecher, T. P., \& Donn, B. 1965, ApJ, 142, 1681

Steglich, M., Jäger, C., Rouillé, G., et al. 2010, ApJ, 712, 16

Steglich, M., Bouwman, J., Huisken, F., \& Henning, T. 2011, ApJ, 742, 2

Tielens, A. G. G. M. 2008, ARA\&A, 46, 289

Uchida, K. I., Sellgren, K., Werner, M. W., \& Houdashelt, M. L. 2000, ApJ, 530,817

Valencic, L. A., Clayton, G. C., \& Gordon, K. D. 2004, ApJ, 616, 912

Van Diedenhoven, B., Peeters, E., Van Kerckhoven, C., et al. 2004, ApJ, 611, 928

Zubko, V., Dwek, E., \& Arendt, R. G. 2004, ApJS, 152, 211 